\title{
A self-correcting quadrature voltage controlled oscillator
}

\author{
Tomoyuki Arai ${ }^{1,2 a)}$ and Ali Hajimiri ${ }^{2}$ \\ ${ }^{1}$ Fujitsu Laboratories Ltd., \\ 10-1 Morinosato-Wakamiya, Atsugi, Kanagawa 243-0197, Japan \\ ${ }^{2}$ California Institute of Technology, \\ 1200 East California Blvd. Pasadena, California 91125, USA \\ a) arai.tomoyuki@jp.fujitsu.com
}

\begin{abstract}
A self-correcting quadrature voltage controlled oscillator (QVCO) with phase correcting loop is proposed. It comprises the QVCO core and phase correcting loop, which corrects the quadrature phase error. Two LC VCOs, buffers, and phase shifters are coupled in circular configuration to achieve IQ symmetry. This paper introduces the idea of realizing QVCO with low phase noise and accurate quadrature phase by using the phase correcting loop. The simulation results based on the $65 \mathrm{~nm}$ CMOS process show that the self-correcting QVCO has a phase error less than $0.5^{\circ}$ and $1 \mathrm{MHz}$ offset phase noise of $-120 \mathrm{dBc} / \mathrm{Hz}$ at $3.7 \mathrm{GHz}$ with $49 \%$ tuning range.
\end{abstract}

Keywords: CMOS, voltage controlled oscillator (VCO), quadrature, phase error, phase noise

Classification: Integrated circuits

\section{References}

[1] M. Simon: IEEE RFIC Dig. Papers (2004) 431. DOI:10.1109/RFIC.2004.1320644

[2] E. Gotz: ESSCIRC Proc. (2003) 217.

[3] J. Van der Tang and D. Kasperkovitz: ISSCC Dig. Tech Papers (1997) 88. DOI:10. 1109/ISSCC.1997.585273

[4] J. Van der Tang: CICC (2001) 323.

[5] S. Toso, A. Bevilacqua, M. Tiebout, N. Dalt, A. Gerosa and A. Neviani: IEEE J. Solid-State Circuits 45 (2010) 1295. DOI:10.1109/JSSC.2010.2049457

[6] A. Rofougaran, J. Rael, M. Rofougaran and A. Abidi: ISSCC Dig. Tech Papers (1996) 393. DOI:10.1109/ISSCC.1996.488731

[7] S. Li, I. Kipnis and M. Ismail: IEEE J. Solid-State Circuits 38 (2003) 1626. DOI: $10.1109 /$ JSSC.2003.817258

[8] G. Cusmai, M. Repossi, G. Albasini, A. Mazzanti and F. Svelto: IEEE J. SolidState Circuits 42 (2007) 2870. DOI:10.1109/JSSC.2007.908727

[9] A. Mazzanti and P. Andreani: IEEE J. Solid-State Circuits 56 (2009) 2173.

[10] A. Mazzanti, F. Svelto and P. Andreani: IEEE J. Solid-State Circuits 41 (2006) 1305. DOI:10.1109/JSSC.2006.874333

[11] P. Andreani and X. Wang: IEEE J. Solid-State Circuits 39 (2004) 1883. DOI:10. 1109/JSSC.2004.835828

[12] D. Leenaerts, C. Dijkmans and M. Thompson: RFIC (2002) MO2C-3 67. DOI:10. 1109/RFIC.2002.1011512

[13] A. D. Berny, A. M. Niknejad and R. G. Meyer: IEEE J. Solid-State Circuits 40 
(2005) 909. DOI:10.1109/JSSC.2004.842851

[14] L. Romano, S. Levantino, A. Bonfanti, C. Samori and A. L. Lacaita: ISCAS Proc. (2004) 1.161. DOI:10.1109/ISCAS.2004.1328156

[15] A. Tanabe, K. Hijioka, H. Nagase and Y. Hayashi: RFIC (2009) RMO3D-4 263. DOI:10.1109/RFIC.2009.5135536

\section{Introduction}

In-phase and quadrature phase local signals are indispensable to wireless transceivers for modern wireless communication standards, such as WiMAX, IEEE $802.11 \mathrm{ac} / \mathrm{ad}$, and LTE. Moreover, these standards require quadrature signal with low phase noise and accurate quadrature phase to cover higher and wider range of frequency for higher data rate wireless communication. The quadrature signal is generated in many ways, such as using frequency dividers [1, 2], even stage ring oscillators [3, 4], polyphase filters, injection locking [5] and quadrature voltage controlled oscillators (QVCOs) $[6,7,8,9,10,11,12,13,14,15]$. To generate local signals higher than $5 \mathrm{GHz}$ for high data rate standards, polyphase filters are not suitable owing to the passive components variation in the fabrication process and the signal amplitude loss. Frequency dividers are common for generating the quadrature signal; however, they require double quadrature local signal frequency. Furthermore, the buffer amplifiers for frequency dividers also need to operate at two times the necessary frequency, which leads to higher current drain consumption. Though QVCO requires twice of the differential VCO chip area, QVCOs are ideal to generate quadrature local signals, in terms of power consumption.

The phase noise and phase accuracy of QVCOs was extensively studied $[9,11$, 14]. Conventional QVCOs comprise two LC VCOs with coupling current source, as shown in Fig. 1 [6]. The quadrature phase accuracy is controlled by the coupling factor of the two VCOs and the coupling factor is controlled by the coupling current source. The higher coupling factor produces a precise quadrature phase, whereas it deteriorates the phase noise of the oscillation signal $[8,9,10,11,12,14]$, because the extra noise source associated with the coupling current source is added to the oscillator. To overcome the tradeoff between phase noise and quadrature phase accuracy, a self-correcting QVCO is proposed.

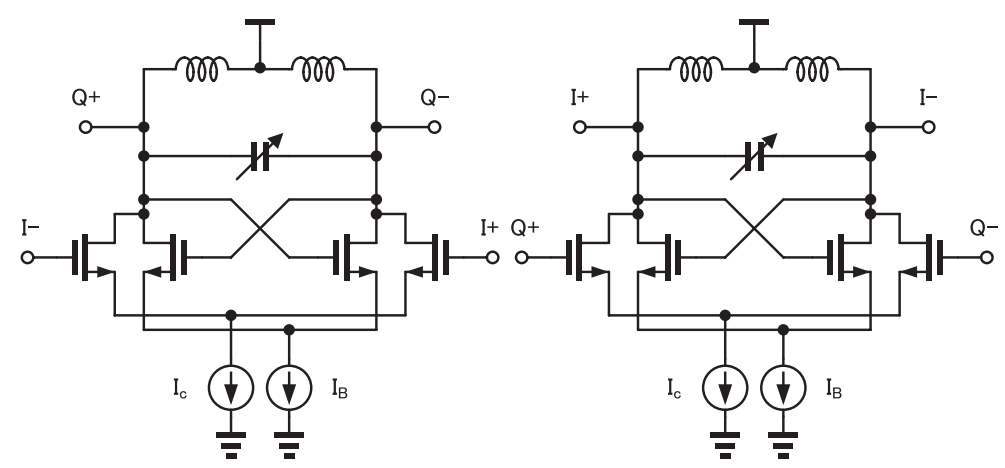

Fig. 1. Topology of a conventional QVCO 


\section{Self-correcting QVCO}

A self-correcting loop shown in Fig. 2 is introduced to the QVCO. The loop corrects the quadrature signal phase error. The self-correcting QVCO comprises the QVCO core and quadrature phase correcting loop (Fig. 2(a)). The QVCO core consists of two LC VCOs, VCO output buffers, and phase shifters. The phase correcting loop includes buffers, phase detectors, and low-pass filters. The differential IQ signal from the QVCO core is connected to the buffers of the loop and their output is fed to the phase detector. The phase detector generates dc output components proportional to the quadrature phase error and high-frequency components, which are then removed by low-pass filters. In addition, the phase shifter controls the phase of the QVCO core IQ signal. The coupling technique of two LC VCOs in the core reduces the noise source of the oscillator compared to conventional QVCO, which requires extra current source, because no extra current and transistors are required. Fig. 2(b) shows the QVCO core block diagram. All the components are configured in a symmetric manner in terms of the IQ signal, as shown in the diagram. Thus, the symmetric layout is necessary to realize an accurate quadrature phase. The core includes buffers that are used as the output buffer of the VCOs. These buffers are necessary to isolate the effect of the VCO load. Therefore, the use of buffers does not increase the current consumption of the QVCO. The I and Q output signals come out at the nodes between the two buffers.

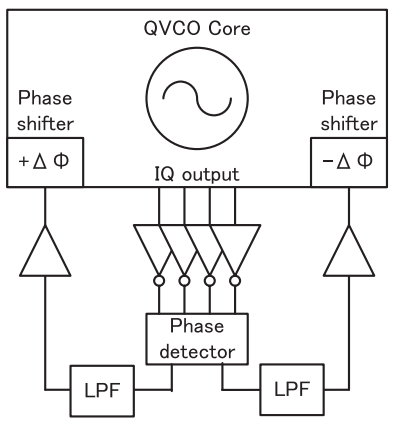

(a)

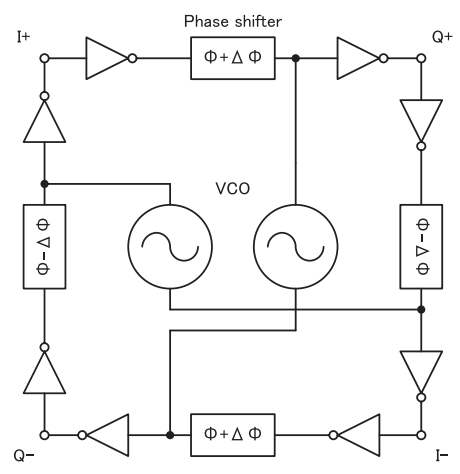

(b)

Fig. 2. (a) Self-correcting QVCO, (b) QVCO core.

\section{Phase correcting loop}

The phase correcting loop compensates for the quadrature phase error owing to the inductor coupling, device mismatch [10], and asymmetric parasitic components.

The block diagram of the phase correcting loop is shown In Fig. 3. $\Phi_{\mathrm{eIN}}$ and $\Phi_{\text {eOUT }}$ are the quadrature phase errors at the input and output of the phase correcting loop. The loop consists of a phase detector, a low-pass filter, an amplifier, and a phase shifter and their corresponding outputs are $V_{1}, V_{2}, V_{3}$, and $\Delta \varphi$. The phase detector detects the error of the IQ signal and the low-pass filter removes the high-frequency components. The dc component of the IQ phase error is used to control the phase of the IQ signal with the phase shifter. 


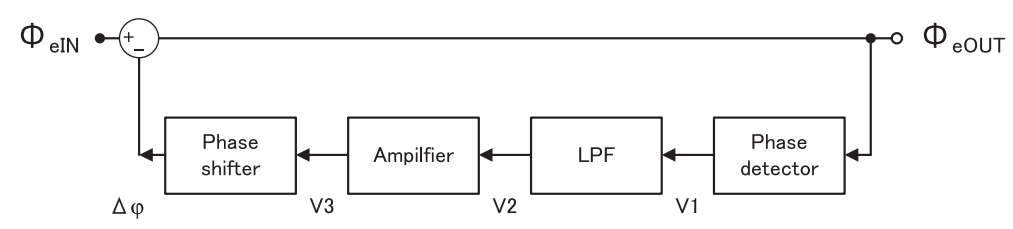

Fig. 3. Block diagram of the phase correcting loop

\subsection{Phase detector}

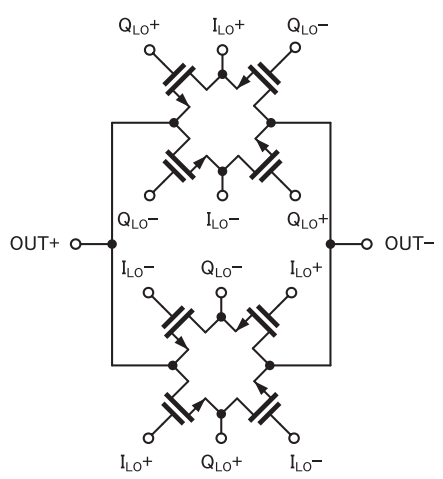

Fig. 4. Schematic of phase detector

Fig. 4 shows the phase detector. Passive mixers receive differential IQ signals from the QVCO core and have differential outputs. Because the phase detector mixes the IQ local signals, the output includes a dc component and two times the local signal frequency. The output signal $\mathrm{V}_{1}$ is given by

$$
\mathrm{V}_{1}(\mathrm{t})=4 \cdot \mathrm{A}_{\mathrm{VCO}} \cdot \sin \left(2 \cdot \omega_{0} \cdot \mathrm{t}+\varphi_{\mathrm{e}}(\mathrm{t})\right)+4 \cdot \mathrm{A}_{\mathrm{VCO}} \cdot \sin \left(\varphi_{\mathrm{e}}(\mathrm{t})\right)
$$

where $\mathrm{A}_{\mathrm{VCO}}$ is the amplitude of the QVCO output signal, and $\varphi_{\mathrm{e}}$ is the quadrature phase error. As the $2 \cdot \omega_{0} \cdot t$ component is removed by the low pass filter, the transfer function is approximated as

$$
\mathrm{H}_{\mathrm{PD}}(\mathrm{s})=\frac{\mathrm{V}_{1}(\mathrm{~s})}{\varphi_{\mathrm{e}}(\mathrm{s})}=4 \cdot \mathrm{A}_{\mathrm{VCO}}
$$

\subsection{Low-pass filter}

Resistors and capacitors are used to design the low-pass filter. The latter attenuates two times the QVCO oscillation frequency component of the phase detector output; hence, the dc component owing to the IQ phase error is the output of the low-pass filter.

\subsection{Amplifier}

The phase correcting loop uses operational amplifiers to adjust the loop gain of the phase correcting loop. It is designed as a narrow-bandwidth low-power amplifier, as it only needs to increase the amplitude of the dc component of the phase detector output. It consumes $0.5 \mathrm{~mW}$ from the $1.0 \mathrm{~V}$ power supply.

\subsection{Phase shifter}

Fig. 5 shows the phase shifter, which consists of resistors, capacitances, varactor diodes, and transistor switches. The phase shift is controlled by changing the 


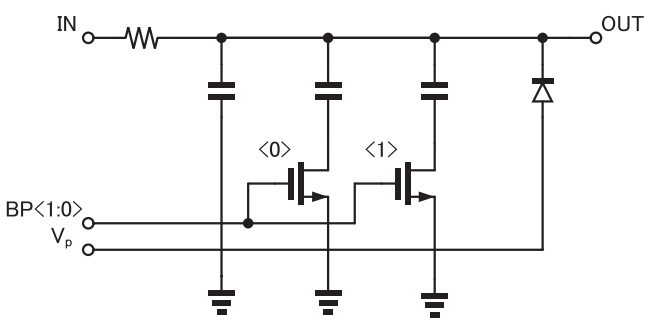

Fig. 5. Schematic of phase shifter

capacitance value with a 2-bit switch and the varactor control voltage applied at node Vp (Fig. 5). The capacitance value is changed based on the IQ local signal frequency.

The transfer function of the phase shifter is expressed as

$$
\mathrm{H}_{\mathrm{PS}}(\mathrm{s})=\frac{\varphi(\mathrm{s})}{\mathrm{V}_{3}(\mathrm{~s})}=\frac{\mathrm{K}_{\mathrm{PS}}}{\mathrm{s}}
$$

where $K_{P S}$ is the coefficient of the transfer function.

\subsection{Phase correcting loop}

The open-loop transfer function $\mathrm{G}_{\mathrm{OP}}$ is expressed as

$$
\begin{array}{r}
\mathrm{G}_{\mathrm{OP}}(\mathrm{s})=\mathrm{H}_{\mathrm{PD}}(\mathrm{s}) \cdot \mathrm{H}_{\mathrm{LPF}}(\mathrm{s}) \cdot \mathrm{H}_{\mathrm{AMP}}(\mathrm{s}) \cdot \mathrm{H}_{\mathrm{PS}}(\mathrm{s}) \\
=\frac{4 \cdot \mathrm{A}_{\mathrm{VCO}}}{\left(1+\mathrm{R}_{\mathrm{LPF}} \mathrm{C}_{\mathrm{LPF}}\right)\left(\frac{\mathrm{a}_{0}}{1+\mathrm{b}_{1} \cdot \mathrm{s}}\right)^{2}\left(\frac{\mathrm{K}_{\mathrm{PS}}}{\mathrm{s}}\right)}
\end{array}
$$

where $\mathrm{R}_{\mathrm{LPF}}$ and $\mathrm{C}_{\mathrm{LPF}}$ are the resistance and capacitance of the low-pass filter, $\mathrm{a}_{0}$ and $b_{1}$ are the coefficients of the amplifier transfer function. Therefore, the close-loop transfer function $\mathrm{G}_{\mathrm{CL}}(\mathrm{s})$ results in

$$
\mathrm{G}_{\mathrm{CL}(\mathrm{s})}=\frac{\varphi_{\mathrm{eOUT}}(\mathrm{s})}{\varphi_{\mathrm{eIN}}(\mathrm{s})}=\frac{1}{1+\mathrm{G}_{\mathrm{OP}(\mathrm{s})}}
$$

The phase error response was analyzed on the basis of the closed-loop transfer function. Fig. 6(a) shows the phase error response in the initial phase error of the $1^{\circ}$ case. The figure shows the three different gain setups of the amplifier. The gain settings are by default $-6 \mathrm{~dB}$ and $+6 \mathrm{~dB}$ compared to the default gain. The default gain case shows that the initial phase error of $1^{\circ}$ decreased to $0.1^{\circ}$. The figure also shows that the phase error is small, as the loop gain increases. Figs. 6(b), (c), and (d) show the impulse response of the phase correcting loop depending on the amplifier gain, output capacitance of the amplifier, and low-pass filter capacitance. Fig. 6(b) shows the three cases of the amplifier gain, default gain, and $+12 \mathrm{~dB}$ and $-12 \mathrm{~dB}$ gain compared to the default; furthermore, it shows the range of the optimum loop gain and that the high gain takes longer to converge. The amplifier output capacitance dependence was examined as shown in Fig. 6(c). It has $15 \mathrm{fF}$, $1 \mathrm{pF}$, and $3 \mathrm{pF}$ impulse response results, and the $3 \mathrm{pF}$ case takes 3 times to converge compared to $15 \mathrm{fF}$ case. This means that the excess capacitance at the output node of the amplifier makes the phase correcting loop unstable. The capacitance of the low-pass filter is also examined in Fig. 6(d) and the results suggest that the $700 \mathrm{fF}$ shows the fastest convergence. It shows that the larger capacitance makes the loop 


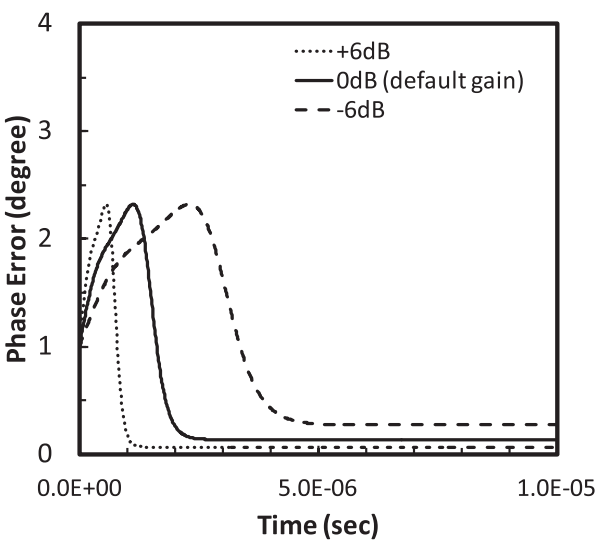

(a)

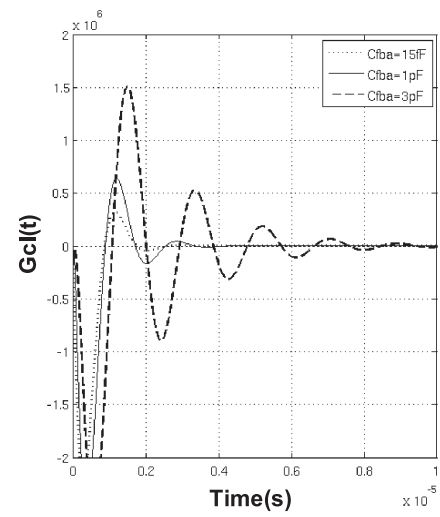

(c)

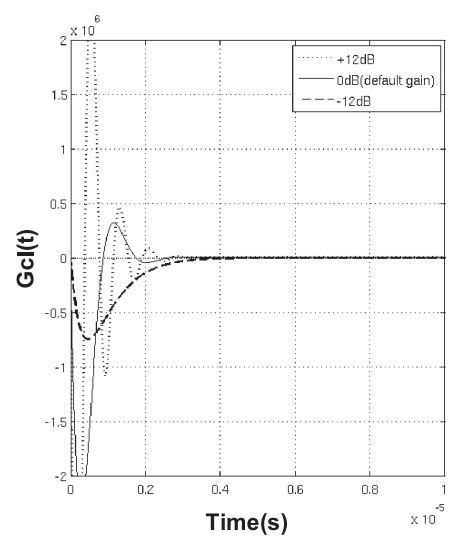

(b)

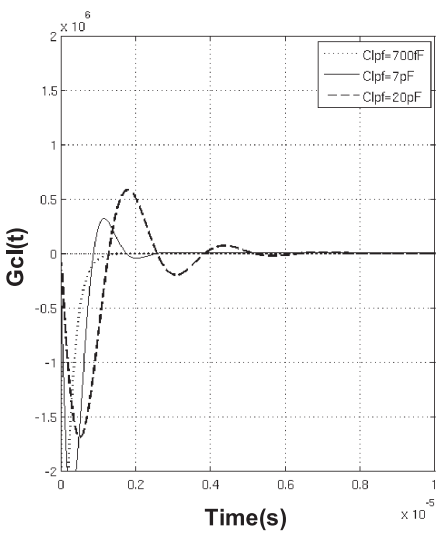

(d)

Fig. 6. Phase correcting loop characteristics: (a) phase error compensation characteristic, (b), (c) and (d) are impulse response of the loop, (b) amplifier gain dependence, (c) amplifier output capacitance dependence, and (d) low-pass filter capacitance dependence.

bandwidth small and slow loop response. The large capacitance makes the low-pass filter bandwidth small and attenuates twice as much of the QVCO oscillation frequency signal. The results show that the accuracy of the phase correcting loop and the loop response have a tradeoff relation.

\section{Phase accuracy and phase noise}

The self-correcting QVCO is designed following the $65 \mathrm{~nm}$ CMOS process with eight aluminum metal layers and a $3 \mu \mathrm{m}$ copper thick metal option.

Based on the loop analysis and QVCO topology, a self-correcting QVCO was designed. The QVCO comprises two LC VCOs as shown in Fig. 7. The transconductance of the differential VCO is generated by the PMOS transistors, and the frequency tuning is realized by the MOM capacitance and the varactor diode. The varactor is controlled by $\mathrm{BV}\langle 1: 0\rangle$ bits to keep the $\mathrm{VCO}$ gain constant over the frequency tuning range and the MOM is used for coarse frequency tuning with $\mathrm{BC}\langle 4: 0\rangle$ bits.

The layout of the QVCO is shown in the Fig. 8. The QVCO occupies the area of $0.7 \mathrm{~mm} \times 0.3 \mathrm{~mm}$ (Fig. $8(\mathrm{a})$ ). The coupling circuits, composed of phase detector, 


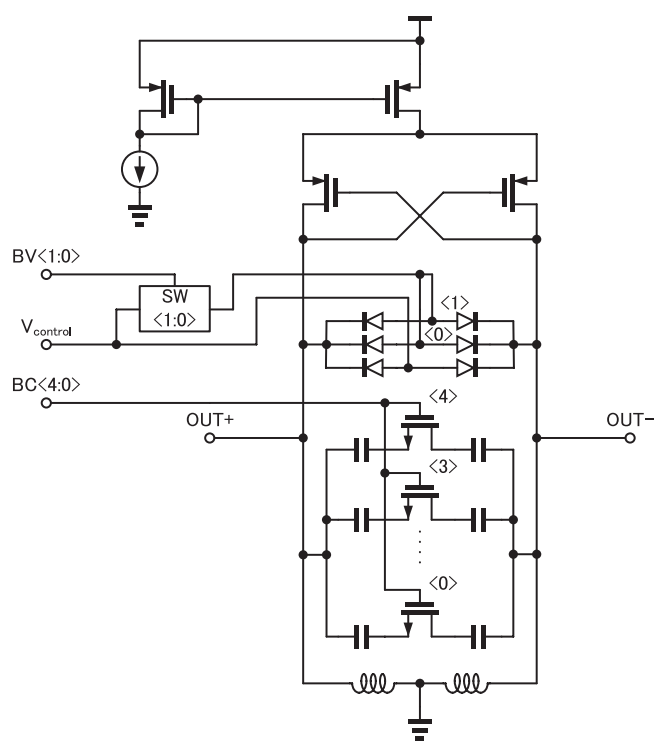

Fig. 7. Schematic of the LC VCO

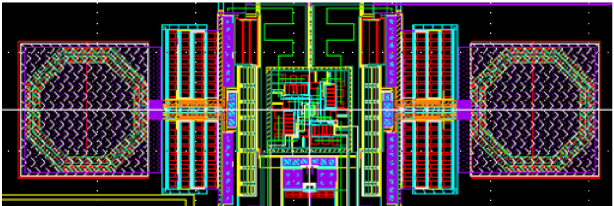

(a)

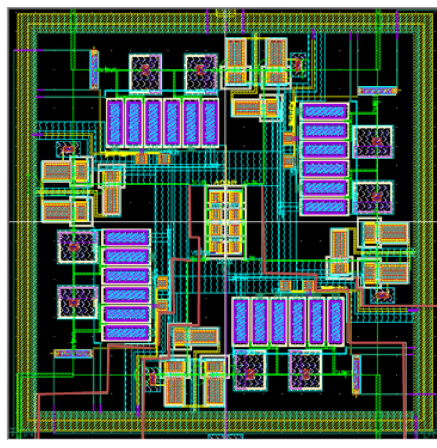

(b)

Fig. 8. Layout of the QVCO (a) QVCO (b) Coupling circuit

low pass filters, buffers, and phase shifters, are in the circular configuration between two LC VCOs to achieve symmetry (Fig. 8(b)).

The settling time of less than $5 \mu$ s, which is $10 \%$ of the WiMAX settling time requirement, is aimed for the phase correcting loop design. Therefore, the loop bandwidth is selected to $1 \mathrm{MHz}$. The settling time and the phase margin of the designed phase correcting loop is about $1 \mu \mathrm{s}$ and $105^{\circ}$, respectively. And the noise contribution to the QVCO output signal is mainly from the transistors of the coupling buffers or the LC VCOs.

The phase correction characteristic of the phase correcting loop based on the QVCO post layout netlist is shown in Fig. 9. The figure shows that the relation between the initial quadrature phase error and the output phase error. Without the phase correcting loop, the initial phase error and the output phase error are the same. The phase correcting loop corrects phase error to less than $0.1^{\circ}$ at the initial phase error of $1^{\circ}$. The total power consumption of the phase correcting loop is $0.5 \mathrm{~mW}$ from the $1 \mathrm{~V}$ supply provided by the amplifiers.

The simulated phase noise at 3.7 and $6.9 \mathrm{GHz}$ at the $1 \mathrm{MHz}$ offset of the selfcorrecting QVCO is -120 and $-110 \mathrm{dBc} / \mathrm{Hz}$, respectively. The frequency tuning 


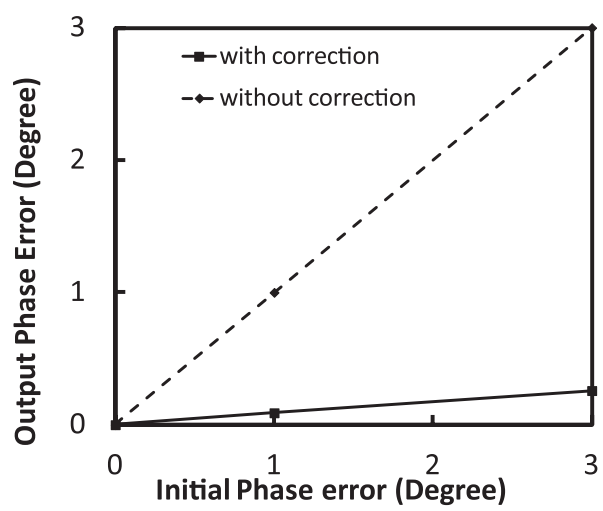

Fig. 9. Phase correction

range is 3.7 to $6.9 \mathrm{GHz}$ and the total power consumption of the self-correcting QVCO is $17 \mathrm{~mW}$ from the $1 \mathrm{~V}$ supply. The self-correcting quadrature VCO performance is compared with state-of-the-art quadrature oscillators in Table I. The following figure of merit has been used for the comparison,

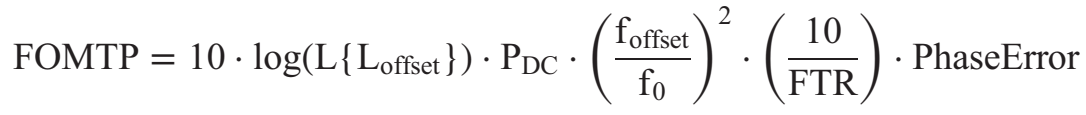

where $L\left\{f_{\text {offset }}\right\}(\mathrm{dBc} / \mathrm{Hz})$ is the phase noise at the $1 \mathrm{MHz}$ offset $\left(\mathrm{f}_{\text {offset }}\right), \mathrm{P}_{\mathrm{DC}}(\mathrm{mW})$ is the dissipated power of the oscillator, $\mathrm{f}_{0}$ is the oscillation frequency, FTR (\%) is the frequency tuning range of the oscillator, and the PhaseError $\left({ }^{\circ}\right)$ is the IQ phase error of the quadrature oscillator.

Table I. QVCO performance

\begin{tabular}{|c|c|c|c|c|c|c|}
\hline Ref. & $\begin{array}{c}\text { Power } \\
{[\mathrm{mW}]}\end{array}$ & $\begin{array}{c}\text { Center } \\
\text { freq. } \\
{[\mathrm{GHz}]}\end{array}$ & $\begin{array}{c}\text { Tuning } \\
\text { range } \\
{[\%]}\end{array}$ & $\begin{array}{c}\text { Phase } \\
\text { noise } \\
{[\mathrm{dBc} / \mathrm{Hz}]}\end{array}$ & $\begin{array}{c}\text { Phase } \\
\text { Error } \\
{\left[{ }^{\circ}\right]}\end{array}$ & $\begin{array}{c}\text { FOMTP } \\
{[\mathrm{dBc}]}\end{array}$ \\
\hline 2 & 12 & 5.3 & 78 & -102 & 1.5 & -185 \\
\hline 7 & 7.2 & 2.6 & 15 & -115 & 1.0 & -177 \\
\hline 8 & 32 & 1.9 & 11 & -131 & 1.0 & -181 \\
\hline 9 & 2.8 & 5.0 & 5 & -103 & 1.3 & -166 \\
\hline $\begin{array}{c}\text { This work } \\
\text { post SIM }\end{array}$ & 17 & 5.3 & 49 & -120 & $<0.5$ & -200 \\
\hline
\end{tabular}

\section{Conclusions}

A self-correcting QVCO with phase correcting loop was proposed. Conventional QVCOs suffer from the tradeoff between quadrature phase accuracy and phase noise. The phase correcting loop corrects the quadrature phase error while maintaining low phase noise. It also consumes little current from the $1.0 \mathrm{~V}$ supply because it mainly comprises passive circuits and does not require extra coupling circuits. Furthermore, the coupling technique of two LC VCOs is characterized by low noise and low power consumption. 